\section{Ocorrência de Aedes aegypti e Aedes albopictus em bromélias cultivadas no Jardim Botânico Municipal de Bauru, São Paulo, Brasil}

Aedes aegypti and Aedes albopictus in bromeliads grown in the Bauru Municipal Botanical Gardens, São Paulo, Brazil

Ocurrencia de Aedes aegypti y Aedes albopictus en bromelias cultivadas en el Jardín Botánico Municipal de Bauru, São Paulo, Brasil
Viviane Camila de Oliveira 1

Luiz Carlos de Almeida Neto 1

doi: 10.1590/0102-311X00071016

\section{Correspondência}

V. C. Oliveira

Jardim Botânico Municipal de Bauru.

Rod. Comandante João Ribeiro de Barros, Km 232, Bauru, SP 17035-245, Brasil.

vivicamila@yahoo.com.br

1 Jardim Botânico Municipal de Bauru, Bauru, Brasil. cando implicações epidemiológicas do uso dessas plantas. A maioria de larvas encontradas foi de mosquitos do gênero Culex, enquanto as de A. aegyptie A. albopictus foi ocasional. O uso de telas para proteção das plantas, a exposição ao sol e a maior quantidade de água no tanque podem ter influenciado a ocorrência e agrupamento das larvas.

Aedes; Bromélias; Vetores de Doenças 


\section{Introdução}

Muitas bromélias acumulam água em um reservatório formado pela disposição de suas folhas, chamado de tanque, criando um micro-hábitat propício à sobrevivência de larvas de mosquitos. Desde o primeiro relato da presença de Aedes (Stegomyia) aegypti (Linnaeus, 1762) em bromélias 1, pesquisadores investigam a possibilidade de que essas plantas possam significar um incremento de criadouros para essa e outras espécies de mosquitos 2 .

As larvas do A. aegypti, principal vetor do vírus da dengue, podem ser encontradas especialmente em criadouros artificiais como garrafas, pneus e vasos de plantas, mas também em axilas de plantas e tanques de bromélias ${ }^{3}$. Já o Aedes (Stegomyia) albopictus (Skuse, 1894), vetor com potencial para a transmissão da dengue e de outras doenças virais como febre chikungunya e zika 4,5 , está melhor adaptado a hábitats preservados, fragmentos florestais e áreas rurais.

O Jardim Botânico Municipal de Bauru, São Paulo, Brasil, abriu em 2014 para visitação pública a sua coleção de bromélias e desde então surgiu a preocupação com a possível ocorrência de larvas de A. aegypti e A. albopictus em tanques de suas bromélias. Em vista disso, este trabalho teve como objetivo determinar a relevância de bromélias do Jardim Botânico Municipal de Bauru como criadouros de larvas de A. aegypti e A. albopictus, destacando implicações epidemiológicas da utilização dessas plantas em coleções de jardins botânicos e para fins paisagísticos.

\section{Material e métodos}

Este estudo foi desenvolvido no Jardim Botânico Municipal de Bauru. O clima da região é Aw (tropical chuvoso com inverno seco) 6 , com temperatura média anual de $23,8^{\circ} \mathrm{C}$ e precipitação média anual de $142,75 \mathrm{~mm}$ 7. O Jardim Botânico Municipal de Bauru fica inserido em um fragmento florestal e sua área de visitação, onde estão alocadas as bromélias utilizadas neste estudo, está distante cerca de um quilômetro de áreas residenciais (Figura 1). Sua coleção de bromélias conta com 392 plantas, alocadas dentro de um viveiro de $300 \mathrm{~m}^{2}$ coberto no teto e nas laterais com tela de sombreamento, irrigadas quinzenalmente. Os gêneros melhores representados são Aechmea, Neoregelia, Nidularium e Vriesea. Além dessas, existem 93 bromélias alocadas em jardins, das espécies Aechmea blanchetiana (Backer) L.B.Sm. e Alcantarea imperialis (Carrière) Harms, expostas a sol pleno e pluviosidade natural. Todas as bromélias estavam distantes, no máximo, 100m entre si.

Foi verificada mensalmente a presença de larvas no tanque e axila de todas as bromélias do Jardim Botânico Municipal de Bauru, no período de janeiro a dezembro de 2015. Quando a presença era constatada, as larvas eram coletadas, contadas, colocadas em frascos de vidro contendo álcool a 70\% e encaminhadas para o Centro de Controle de Zoonoses da Prefeitura Municipal de Bauru, para identificação em nível de gênero ou espécie. Para a coleta de água, utilizou-se uma seringa e um tubo plástico flexível.

\section{Resultados}

Foram realizadas 38 coletas de larvas de um total de 5.820 verificações; dessas coletas, uma foi positiva para $A$. aegypti, cinco para $A$. albopictus e a grande maioria das coletas positivas para larvas de mosquitos do gênero Culex (Tabela 1). Do total de 152 larvas coletadas, 143 foram de larvas de mosquito do gênero Culex, duas de A. aegypti e sete de A. albopictus (Tabela 2).

As larvas foram coletadas durante todo o ano, com exceção apenas do mês de julho, a maioria delas nas axilas e no tanque das bromélias (Tabela 2). Foram coletadas 148 larvas em bromélias localizadas fora do viveiro e apenas quatro larvas em bromélias localizadas dentro do viveiro. A ocorrência de larvas em bromélias localizadas dentro do viveiro foi restrita às do gênero Aechmea, enquanto fora do viveiro foram encontradas larvas em bromélias das duas espécies ocorrentes, Aechmea blanchetiana e Alcantarea imperialis. Foi verificado agrupamento espacial das larvas, fora do viveiro, em duas bromélias da espécie A. imperialis, onde foram coletadas a grande maioria das larvas. Nessas duas bromélias, 


\section{Figura 1}

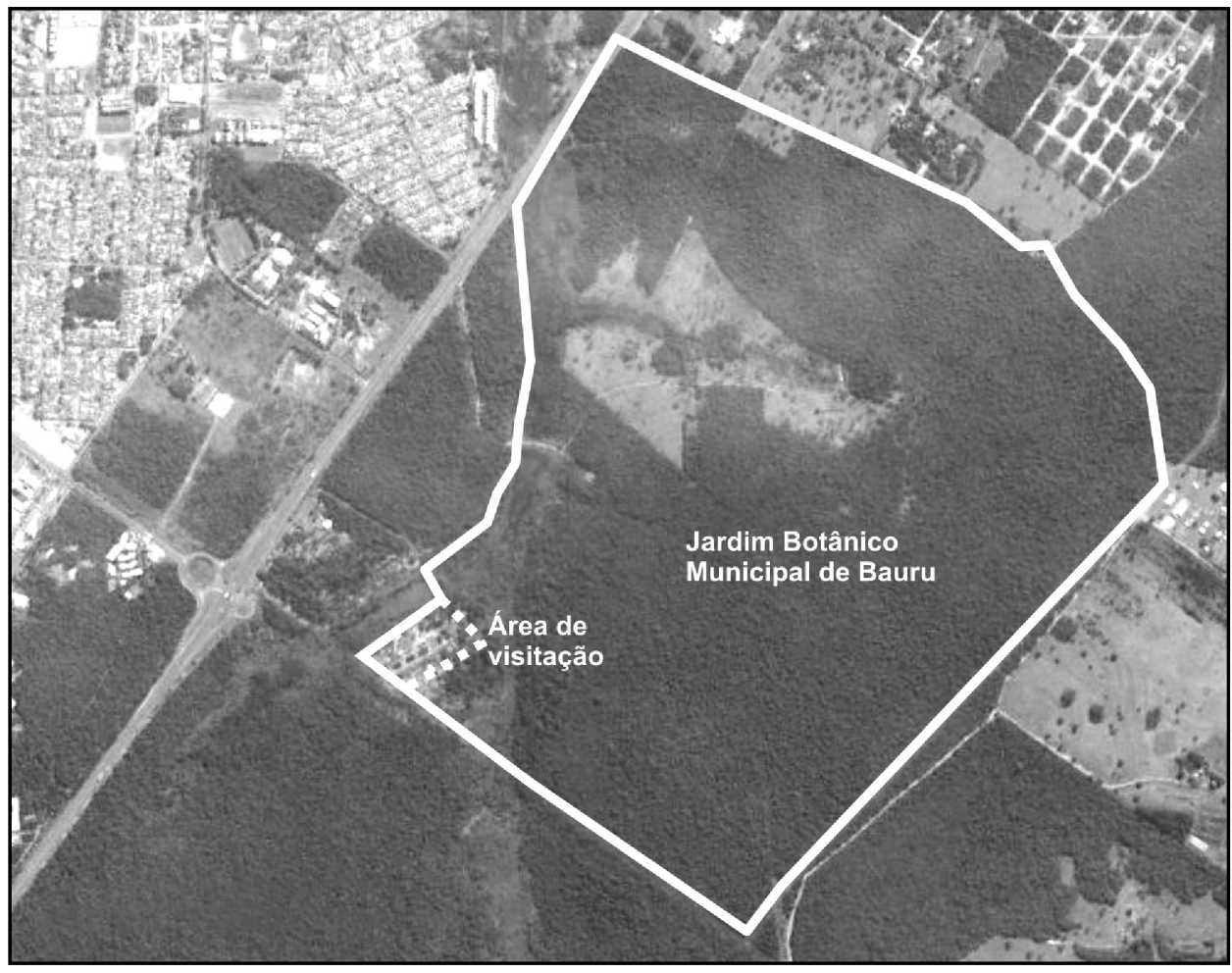

0 $1 \mathrm{~km}$
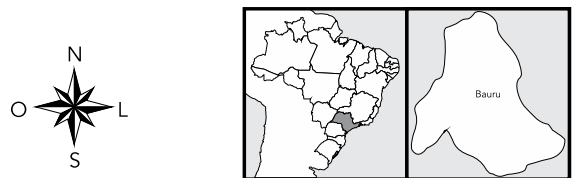

Fonte: Google Earth (https://www.google.com.br/intl/pt-BR/earth/) 2016.

no momento das coletas, foi encontrada uma maior quantidade de água no tanque e axilas, comparada às demais bromélias, bem como maior quantidade de matéria orgânica.

\section{Discussão}

O número de larvas de mosquitos encontrado nas bromélias neste trabalho foi baixo se comparado com estudos realizados em áreas urbanas e periurbanas, que correspondem a maioria dos trabalhos $8,9,10,11$. O baixo número de larvas possivelmente está relacionado à localização do Jardim Botânico Municipal de Bauru, distante cerca de um quilômetro de áreas residenciais e inserido em um grande fragmento florestal, reforçando a predileção de diversas espécies de mosquitos por áreas urbanas.

A prevalência de larvas do gênero Culex foi um resultado satisfatório e pode redirecionar trabalhos de vigilância ambiental, já que a maioria das espécies de Culex não tem importância epidemiológica no Brasil atualmente, pois não oferecem riscos à saúde humana 12. Além disso, pode haver competição de tais larvas com as de outras espécies de importância epidemiológica, como as do Aedes. 
Tabela 1

Coletas de larvas em bromélias alocadas dentro e fora do viveiro de coleções do Jardim Botânico Municipal de Bauru, São Paulo, Brasil, de janeiro a dezembro de 2015

\begin{tabular}{|c|c|c|c|c|}
\hline Local & $\begin{array}{l}\text { Coletas positivas/ } \\
\text { Total de verificações }\end{array}$ & $\begin{array}{c}\text { Coletas positivas para Aedes } \\
\text { aegypti/Total de coletas }\end{array}$ & $\begin{array}{l}\text { Coletas positivas para Aedes } \\
\text { albopictus/total de coletas }\end{array}$ & $\begin{array}{c}\text { Coletas positivas para Culex } \\
\text { sp./Total de coletas }\end{array}$ \\
\hline Dentro do viveiro & $3 / 4.704$ & $0 / 3$ & $1 / 3$ & $2 / 3$ \\
\hline Fora do viveiro & $35 / 1.116$ & $1 / 35$ & $4 / 35$ & $32 / 35$ \\
\hline Total & $38 / 5.820$ & $1 / 38$ & $5 / 38$ & $34 / 38$ \\
\hline
\end{tabular}

Tabela 2

Número de larvas coletadas em bromélias alocadas dentro e fora do viveiro de coleções do Jardim Botânico Municipal de Bauru, São Paulo, Brasil, de janeiro a dezembro de 2015.

\begin{tabular}{|c|c|c|c|c|c|c|c|c|}
\hline & \multicolumn{4}{|c|}{ Dentro do viveiro } & \multicolumn{4}{|c|}{ Fora do viveiro } \\
\hline & Culex sp. & $\begin{array}{l}\text { Aedes } \\
\text { aegypti }\end{array}$ & $\begin{array}{c}\text { Aedes } \\
\text { albopictus }\end{array}$ & Total & Culex sp. & $\begin{array}{c}\text { Aedes } \\
\text { aegypti }\end{array}$ & $\begin{array}{c}\text { Aedes } \\
\text { albopictus }\end{array}$ & Total \\
\hline Janeiro & - & - & - & - & 13 & - & - & 13 \\
\hline Fevereiro & - & - & - & - & 14 & - & - & 14 \\
\hline Março & 2 & - & 2 & 4 & 14 & - & 2 & 16 \\
\hline Abril & - & - & - & - & 51 & 2 & 1 & 54 \\
\hline Maio & - & - & - & - & 3 & - & - & 3 \\
\hline Junho & - & - & - & - & 7 & - & - & 7 \\
\hline Julho & - & - & - & - & - & - & - & - \\
\hline Agosto & - & - & - & - & 14 & - & 2 & 16 \\
\hline Setembro & - & - & - & - & 8 & - & - & 8 \\
\hline Outubro & - & - & - & - & 2 & - & - & 2 \\
\hline Novembro & - & - & - & - & 7 & - & - & 7 \\
\hline Dezembro & - & - & - & - & 8 & - & - & 8 \\
\hline Total & 2 & - & 2 & 4 & 141 & 2 & 5 & 148 \\
\hline
\end{tabular}

O número de larvas de A. aegypti e A. albopictus em bromélias, neste trabalho, também foi muito pequeno se comparado ao número total de observações feitas, podendo-se dizer que esse encontro foi ocasional. A macrobiota existente nesses reservatórios, que inclui predadores naturais de mosquitos, tem um papel importante no controle natural do Aedes 13,14, além da competição interespecífica entre as larvas do Aedes e de outras espécies de mosquitos mais adaptadas a este hábitat 13,14,15. Em bromélias localizadas em ambiente urbano, contudo tais condições podem não estar presentes, permitindo assim a procriação de larvas do Aedes. De acordo com Marques et al. ${ }^{9}$, se comparadas a criadouros artificiais encontrados em áreas urbanas, as bromélias são consideradas secundárias, mas merecem atenção por conta de sua crescente utilização para fins ornamentais.

Um dos fatores que determinou a menor ocorrência de larvas nas bromélias localizadas dentro do viveiro, neste trabalho, pode ter sido a tela de sombreamento que cobre o viveiro, que funcionou como uma barreira mecânica à entrada de mosquitos adultos. Isso sugere uma aplicação prática para a gestão de vetores no que se refere ao uso dessas plantas em coleções, sobretudo em ambientes urbanos, seja por jardins botânicos, colecionadores e cultivadores particulares. Já a preferência dos mosquitos na utilização de reservatórios das bromélias do gênero Alcantarea, verificada neste trabalho, 
possivelmente está associada à maior capacidade de armazenar água apresentada por indivíduos dessa espécie, o que favorece a oviposição e o agrupamento das larvas. Diversos trabalhos propuseram que reservatórios maiores desempenham papel importante no agrupamento de larvas do Aedes 9,16,17 e, por isso, bromélias de gêneros com capacidade de acumular grandes quantidades de água, particularmente em áreas urbanas, necessitam de maior vigilância epidemiológica. A exposição das bromélias ao sol e as diferenças nas características do conteúdo aquático também podem ter influenciado a distribuição das larvas de mosquito, como proposto por Cardoso et al. 17 .

Conclui-se que: (1) neste estudo, as bromélias não constituíram focos do A. aegypti ou A. albopictus, prevalecendo o encontro de larvas de mosquito do gênero Culex; (2) a localização do Jardim Botânico, distante de áreas residenciais, pode ter contribuído para a limitada ocorrência de larvas de mosquito em bromélias; (3) o uso de telas, a exposição das plantas ao sol e a maior capacidade de acumular água de algumas plantas podem ter sido fatores determinantes na distribuição e agrupamento das larvas; (4) em áreas urbanas, com a redução de criadouros artificias, as bromélias podem servir de criadouros alternativos para o A. aegypti ou A. albopictus, por isso a necessidade de manter o monitoramento entomológico de tais plantas.

\section{Colaboradores}

Ambos os autores tiveram contribuições substanciais para a concepção do trabalho; a aquisição, análise e interpretação de dados; elaboração do trabalho e revisão crítica do conteúdo intelectual; aprovação final da versão a ser publicada.

\section{Agradecimentos}

Os autores agradecem ao Centro de Controle de Zoonoses da Prefeitura Municipal de Bauru pela identificação das larvas de mosquitos. 


\section{Referências}

1. Peryassú AG. Os culicídeos do Brasil. Rio de Janeiro: Typographia Leuzinger; 1908.

2. Santos CB, Leite GR, Falqueto A. Does native bromeliads represent importante breeding sites for Aedes aegypti (L.) (Diptera: Culicidae) in urbanized areas? Neotrop Entomol 2011; 40:278-81.

3. Braga IA, Valle D. Aedes aegypti: histórico do controle no Brasil. Epidemiol Serv Saúde 2007; 16:113-8.

4. Martins VP, Silveira DA, Ramalho IL, Florindo MI. Aedes albopictus no Brasil: aspectos ecológicos e riscos de transmissão da dengue. Entomotropica 2013; 28:75-86.

5. Azevedo RSS, Oliveira CS, Vasconcelos PFC. Risco do chikungunya para o Brasil. Rev Saúde Pública 2015; 49:58.

6. Peel MC, Finlayson BL, McMahon TA. Update world map of the Köppen-Geiger climate classification. Hydrology and Earth Systems Sciences 2007; 11:1633-44.

7. IPMet - Centro de Meteorologia de Bauru. Estação meteorológica automática. http://www.ipmet.unesp.br/index 2.php?menu_esq $1=$ \&abre $=$ ip met_html/estacao/historico.php (acessado em 12/ Abr/2016).

8. Forattini OP, Marques GRAM, Kakitani I, Brito M, Sallum MAM. Significado epidemiológico dos criadouros de Aedes albopictus em bromélias. Rev Saúde Pública 1998; 32:186-8.

9. Marques GRAM, Santos RLC, Forattini OP. Aedes albopictus em bromélias de ambiente antrópico no estado de São Paulo, Brasil. Rev Saúde Pública 2001; 35:243-8.

10. Gonçalves KS, Messias MC. Ocorrência de Aedes (Stegomyia) aegypti (Linnaeus, 1762) (Insecta, Diptera, Culicidae) em bromélias, no município do Rio de Janeiro (Rio de Janeiro, Brasil). Biota Neotrop (Online, Ed. Port.) 2008; 8:235-7.

11. Moncellin MG, Simões TC, Silva do Nascimento TF, Teixeira MLF, Lounibos LP, Lourenço de Oliveira R. Bromeliad-inhabiting mosquitoes in na urban botanical Garden of dengue endemic Rio de Janeiro. Are bromeliads productive habitats for the invasive vectors Aedes aegypti and Aedes albopictus? Mem Inst Oswaldo Cruz 2009; 104:1171-6.
12. Consoli RAGB, Oliveira RL. Principais mosquitos de importância sanitária no Brasil. Rio de Janeiro: Editora Fiocruz; 1994.

13. Armbruster P, Hutchinson RA, Gotgreave P. Factor influencing community structure in a South American tank bromeliad fauna. Oikos 2002; 96:225-34.

14. Braks MAH, Honório NA, Lounibos LP, Lourenço-de-Oliveira R, Juliano AS. Interspecific competition between two invasive species of container mosquitoes, Aedes aegypti and Aedes albopictus (Dipetra: Culicidae), in Brazil. Ann Entomol Soc Am 2004; 97:130-9.

15. Richardson BA. The bromeliad microcosm and the assesment of faunal diversity in a tropical forest. Biotropica 1999; 31:321-36.

16. Schafrick NH, Milbrath MO, Berrocal VJ, Wilson ML, Eisenberg JNS. Spatial clustering of Aedes aegypti related to breeding container characteristics in coastal Ecuador: implications for dengue control. Am J Trop Med Hyg 2013; 89:758-65.

17. Cardoso CAA, Oliveira RL, Codeço CT, Motta MA. Mosquitoes in bromeliads at ground level of the Brazilian Atlantic Forest: the relationship between mosquito fauna, water volume, and plant type. Ann Entomol Soc Am 2015; 108:449-58. 


\section{Abstract}

The aim of this study was to observe the occurrence of mosquito larvae, especially Aedes aegypti and Aedes albopictus, in the tanks and axillae of bromeliads at the Bauru Municipal Botanical Gardens, São Paulo, Brazil, highlighting the epidemiological implications for the use of these plants. The majority of the larvae belonged to mosquitos from genus Culex, with only occasional findings of $\mathrm{A}$. aegypti and $\mathrm{A}$. albopictus. The use of screens for protection of the plants, exposure to sunlight, and larger amounts of water in the tanks may have influenced the occurrence and grouping of larvae.

Aedes; Bromelia; Disease Vectors

\section{Resumen}

El objetivo de este trabajo fue observar la ocurrencia de larvas de mosquitos, en especial las del Aedes aegypti $y$ Aedes albopictus, en el tanque $y$ axilas de bromelias del Jardín Botánico Municipal de Bauru, São Paulo, Brasil, destacando las implicaciones epidemiológicas en el cultivo de esas plantas. La mayoría de larvas encontradas fueron de mosquitos del género Culex, mientras que las de A. aegypti $y$ A. albopictus fueron ocasionales. La utilización de telas para la protección de las plantas, su exposición al sol y la mayor cantidad de agua en el tanque pueden haber influenciado la ocurrencia y agrupamiento de larvas.

Aedes; Bromelia; Vectores de Enfermedades 